\title{
A Parallel Linear Temporal Logic Tableau
}

\author{
John C. M ${ }^{\mathrm{c}}$ Cabe-Dansted, Mark Reynolds \\ School of Computer Science and Software Engineering \\ The University of Western Australia \\ Australia \\ john.mccabe-dansted@uwa.edu.au, \\ mark.reynolds@uwa.edu.au
}

\begin{abstract}
For many applications, we are unable to take full advantage of the potential massive parallelisation offered by supercomputers or cloud computing because it is too hard to work out how to divide up the computation task between processors in such a way to minimise the need for communication. However, a recently developed branch-independent tableaux for the common LTL temporal logic should intuitively be easy to parallelise as each branch can be developed independently. Here we describe a simple technique for partitioning such a tableau such that each partition can be processed independently without need for interprocess communication. We investigate the extent to which this technique improves the performance of the LTL tableau on standard benchmarks and random formulas.
\end{abstract}

Keywords: one-pass, tableaux, cloud, parallel, computation, LTL

\section{Introduction}

Parallelisation is important to exploit modern multi-core computers. There has been considerable interest in the potential of tableaux to be parallelised (see e.g. [19], [4], [1]). For this reason we propose and investigate a simple technique for parallelising tableaux with independent branches.

An embarrassingly easy problem is one that can be easily divided into multiple tasks, particularly when there is no need for communication between those tasks. The one-pass tableau of [1] should be an "embarrassingly parallel problem" as each branch is independent. In principle, we can assign each branch to a different thread, and report "satisfiable" if any of the tasks report that the formula is satisfiable.

Nevertheless it is not axiomatic that improving performance via parallelization is easy. In practice, the tableau may have many branches. Blindly assigning a thread to every branch may flood a local machine. On cloud services firing up as many CPUs as there are branches could be embarrassingly expensive. In any case, moving problems to new CPUs has overhead, and so assigning a branch to a new thread may not even improve performance.

A further problem when parallelising tableaux is that multi-threaded programming can be hard. Since order of execution of parallel task may not be deterministic, it can lead to "Heisenbugs" that are hard to reproduce because they only occur occasionally even given fixed input. Eliminating these bugs can be time consuming, and even if all such bugs have been eliminated, it can be hard to trust the correctness of proofs that depend on the correctness of the implementation. Given a limited amount on time to implement and debug a tableau, it may be wise to add more optimisations rather than implement complex support for multi-threaded computation. The contributions of this paper are:

- to introduce a novel, fast and easy way to manage parallel implementations on suitable tableau reasoners which can make maximum use of the power of multiple processors;

- to demonstrate the speed-up in practice using standard and very accessible cloud based high performance and multi-core facilities;

P. Bouyer, A. Orlandini \& P. San Pietro (Eds.): 8th Symposium on Games, Automata, Logics and Formal Verification (GandALF'17)

EPTCS 256, 2017, pp. 166-179 doi 10.4204/EPTCS.256.12 
- to show that we can predict the performance of the technique on unsatisfiable formulas;

- to give an indication of what sorts of problems benefit most from such an approach; and

- to suggest what role such parallel tableau system may play in combination with a host of very different rival reasoning systems.

In the next section we will quickly review some relevant details of the recently introduced LTL tableaux, which has the one-pass and branch-independence aspects that required for our parallelisation technique. In Section 3, we introduce the parallel algorithm which manages the partitioning of the tableau search into separate jobs for the given number of parallel processors. In Section 4 , we present some benchmarks of satisfiable and unsatisfiable formulas. In Section 5, we consider the shape of tableaux and how this affects when parallelisation is effective. In Section 6, we consider what our parallel implementation adds to the portfolio of reasoners with different strengths. Finally, we present a brief conclusion mentioning future work. Some additional benchmarks are available in the expanded version [8].

\section{A new one-pass embarrassingly parallel LTL tableau}

In this section we give a brief introduction to the branch-independent LTL tableaux system recently introduced in [17] and evaluated experimentally against other state-of-the-art reasoners in [1].

LTL [16], or propositional linear time temporal logic, is a long established formalism for tackling a range of industrially important reasoning tasks from the correctness of hardware systems, through AI planning to specifying work flow. LTL satisfiability checking, seeing if an LTL formula has a model or not, has recently being receiving renewed attention [9] in "sanity checking" of specifications: there is no point trying to implement an inconsistent specification.

An important property of LTL and the leviathan tableau is that they only consider one branch. This is all that our parallelisation technique depends upon. It does not otherwise interact the semantics of LTL; however, for completeness we now introduce the syntax of LTL. LTL adds temporal operators such as tomorrow $X$, eventually $F$, always $G$ and until $U$ on top of classical propositional logic and evaluates truth of formulas along paths of states, where each proposition is true or false at the individual states. We define the

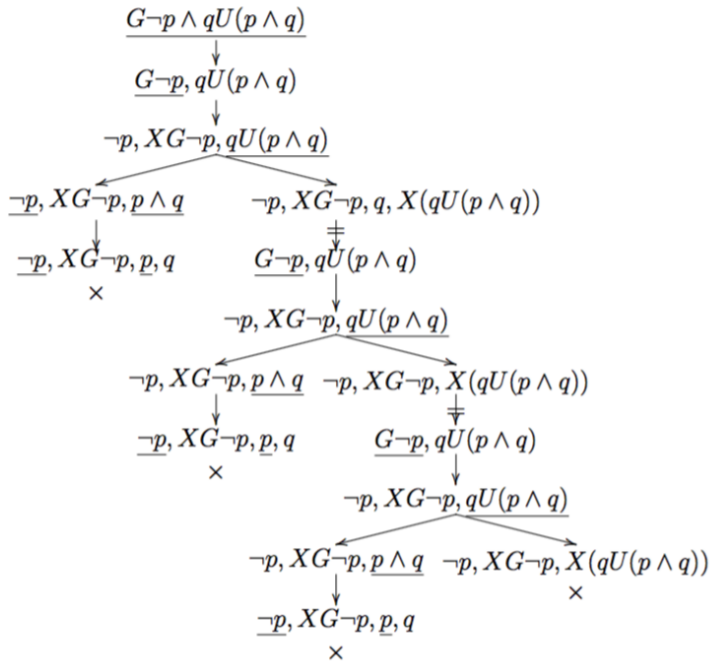

Figure 1: Tableau Example using Prune Rule to close rightmost branch (well-formed) formulas of LTL recursively: any atomic proposition is a formula, if $\alpha$ and $\beta$ are formulas then $\neg \alpha, \alpha \wedge \beta, \alpha \vee \beta, X \alpha, F \alpha, G \alpha$ and $\alpha U \beta$ are all formulas of LTL. See [17] for details of the semantics. We say that a formula is satisfiable iff there is a sequence of states where the formula holds at the initial state when evaluated along the whole path.

There are quite a range of rival reasoning techniques and tools which can be brought to bear on the LTL satisfiability problem. They include tableaux plt1 [19], resolution TRP++ [7], resolution LS4 [20], 
symbolic model verification NuSMV [2], and automata Aalta [11]. Surprisingly (or is it unsurprisingly?), there are no clear overall winners. Therefore, "portfolio" reasoners such as [10] have a part to play: trying a variety of tools in parallel on the same input formula.

As mentioned in the Introduction, apart from the portfolio reasoners and a very preliminary account of an idea in [13], parallel computing techniques have not been applied to LTL. Recently a new tableau rule was introduced that allows a traditional tree-shaped tableau [17]. It is a one-pass tree-shaped one like Schwendimann [19] but unlike Schwendimann's, there is no need for communication between branches.

The new rule, called the Prune rule, can be added to fairly standard tableau construction rules. It is some sort of negative counterpart of the looping rule which allows a branch to be closed successfully if a label is repeated along the branch and cures to all eventualities (required by the label) are witnessed in between the two appearances of the same label. The new rule allows branches to be failed, i.e. closed unsuccessfully, just because they have become repetitious without making progress in witnessing cures to eventualities. The Prune rule can be applied when three occurrences of the same label appear down one branch with no new eventualities being cured between the second and third occurrence.

By using the new Prune rule in amongst quite traditional tree-shaped classical and modal logic tableau construction rules, we can provide a sound and complete tableau system for LTL. The completeness proof is quite intricate. Figure 1 presents a small example tableau. The reader will need to see [17] for full details of the rules, diagram notation and proofs.

A demonstration Java implementation of the tableau system is available at http://staffhome. ecm. uwa.edu. au/ 00054620/research/Online/ltlsattab.html which allows users to understand the tableau building process in a step by step way but it is not designed as a fast implementation. A quite straightforward but fast and efficient $\mathrm{C}++$ implementation of the new tableau written by Matteo Bertello of Udine University is available from https://github.com/Corralx/leviathan. This is evaluated in [1] against a wide range of state-of-the-art reasoners using benchmarks from [18].

The Prune-based tableau is fast, and it is also intuitive and simple to use manually and implement. It has some other advantages including extensibility: for example a clever but simple additional rule allows the tableau to also handle past-time operators [5]. The important aspects of this new pruning-based LTL tableau approach for us are its one-pass nature and the fact that branches can be built and evaluated independently of each other. As noted in [17] this suggests that the tableau search in the new tableau is an "embarrassingly parallel" task [3]. Let us see if we can make use of this potential.

\section{Parallelisation Technique}

We parallise the tableau using another rule we call the Decline rule. The idea is that at some fixed "split_depth", we divide the branches between multiple independant "jobs" that can be run in parallel. The decline rule fails the present branch if it has been assigned to another job. As soon as any job halts and reports that the formula is satisfiable, the entire parallel algorithm halts and reports that the formula is satisfiable. If all jobs have halted and reported that the formula is unsatisfiable, the parallel algorithm halts and reports to the user that the formula is unsatisfiable (if only some jobs report that the formula is unsatisfiable, this may just be because none of the model(s) of the formula are reachable from vertices assigned to those jobs).

The division of a tableau into jobs is illustrated in Figure 2. Parts of the tree above the split_depth are overhead that need to be constructed by all jobs, whereas parts of the tree below split_depth are parallelised. In Figure 2 the jobs have been assigned in a round-robin fashion, and this has resulted in job 1 picking a left branch each time. We also see that this has resulted in more work being assigned to 


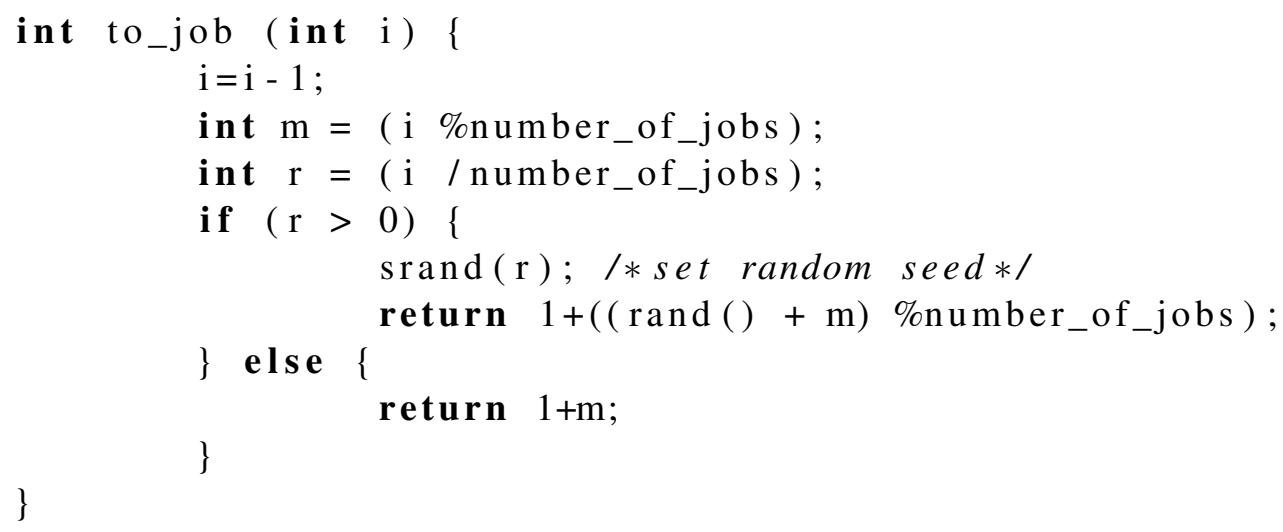

Algorithm 1: to_job: Algorithm to assign the $i^{\text {th }}$ branch of given depth to a job. This algorithm can be informally described as "Where $n$ is the number of jobs, break the jobs into blocks of size $n$. Assign each block of $n$ jobs in a round-robin fashion, but for each block start assigning jobs at some pseudo-randomly chosen job".

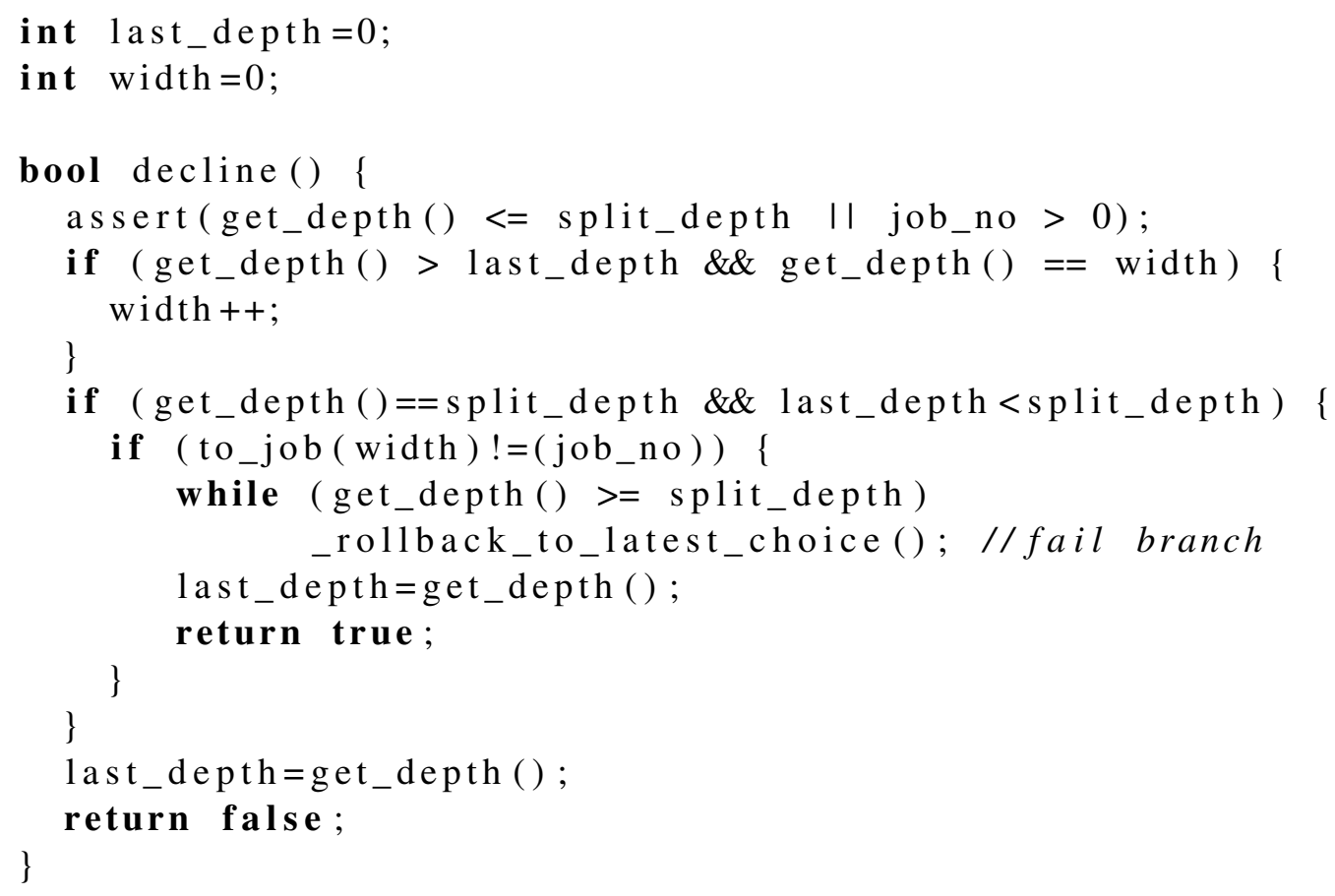

Algorithm 2: The Decline rule. This Algorithm fails/rolls-back the current branch iff we are at split_depth, and the present branch is not assigned to the current job_no. 


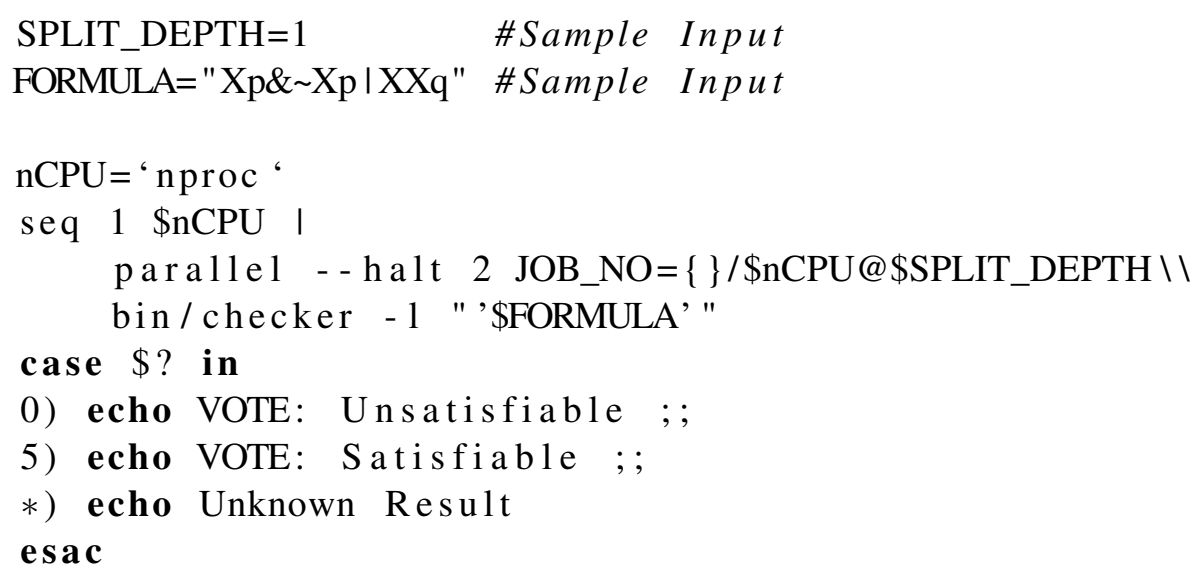

Algorithm 3: Shell script wrapper to run jobs on each CPU of a single machine, bin/checker is the leviathan tableau, parallel --halt 2 runs jobs in parallel until a non-zero exit code is returned, the JOB_NO environment variable has the format job-number/number-of-jobs@split_depth, \$? is the exit code of the last command. This reports satisfiable as soon as any of the jobs reports satisfiable, or unsatisfiable once all jobs report unsatisfiable.

job 1. Although this figure is for illustrative purposes, we have found that using a simple round-robin assignment can lead to an imbalance in the amount of work assigned to each job. For this reason we use Algorithm 1 to assign branches to jobs.

We have not shown the formulas on each branch of Figure 2. This is because this parallisation technique does not take as input formulas, it only works on the shape of the graph. The only property this parallelisation technique requires of the tableau is that the branches are independent.

We see that the algorithms are fairly simple. For example, Algorithm 3 is roughly as lengthy as Algorithms 1 and 2. despite Algorithm 3 being just a wrapper around our modified leviathan and GNU parallel [21]. Indeed Algorithm 3 is a greatly simplified version of the actual script parallel. sh that we use to divide jobs between instances, whereas Algorithms 1 and 2 are very similar to the actual $\mathrm{C}++$ code. Additionally we see that they are not tightly coupled to the imple-

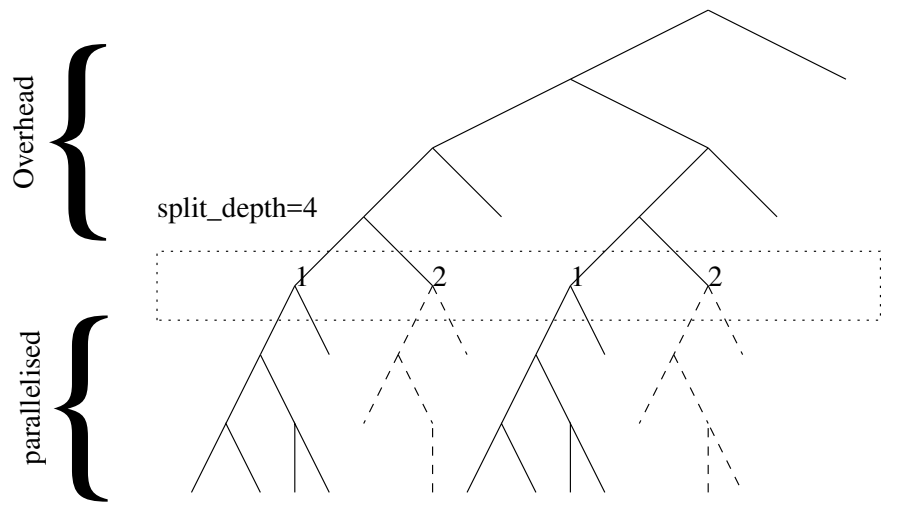

Figure 2: Portion of an example tableau constructed by job 1 out of 2. The portion of the tableau above split_depth is constructed by all jobs. We have labelled vertices at depth split_depth with a job number. At split_depth job $j$ immediately rejects/fails the current branch if it is not labelled with $j$. mentation of leviathan. Only 51 new lines of code were added to the $\mathrm{C}++$ code in total, including instrumentation. This increased the size of the file solver.cpp which implements the tableau algorithm to 608 lines of code in total. The 


\begin{tabular}{|c|c|c|c|c|c|c|c|c|}
\hline & \multicolumn{4}{|c|}{ Time Taken } & & \multicolumn{3}{|c|}{ speedup } \\
\hline Name & $\times 1$ & $\times 2$ & $\times 8$ & $\times 88$ & formula & $\times 2$ & $\times 8$ & $\times 88$ \\
\hline U3_0002 & 27.16 & 13.49 & 3.93 & 0.59 & $\operatorname{trp} / \mathrm{N} 5 \mathrm{x} / 32-5-0-32-3-0-200001$ & 2.01 & 6.91 & 46.03 \\
\hline U3_0003 & 51.51 & 23.51 & 7.52 & 1.01 & $\mathrm{p} / \mathrm{N} 5 \mathrm{x} / 30-5-0-30-3-0-200003$ & 2.19 & 6.85 & 51.00 \\
\hline U3_0004 & 52.39 & 25.50 & 8.54 & 1.06 & $\operatorname{trp} / \mathrm{N} 5 \mathrm{x} / 40-5-0-40-3-0-200000$ & 2.05 & 6.13 & 49.42 \\
\hline U3_0005 & 56.51 & 27.74 & 11.14 & 1.26 & $\operatorname{trp} / \mathrm{N} 5 \mathrm{x} / 32-5-0$ & 2.04 & 5.07 & 44.85 \\
\hline U3_0006 & 74.16 & 36.51 & 12.15 & 1.51 & $3-0-200006$ & 2.03 & 6.10 & 49.11 \\
\hline U3_0007 & 82.79 & 40.70 & 11.61 & 2.05 & $\operatorname{trp} / \mathrm{N} 5 \mathrm{x} / 32-5-0-32-3-0-200009$ & 2.03 & 7.13 & 40.39 \\
\hline U3_0008 & 118.41 & 59.93 & 19.55 & 3.66 & $\operatorname{trp} / \mathrm{N} 5 \mathrm{x} / 30-5-0-30-3-0-200000$ & 1.98 & 6.06 & 32.35 \\
\hline S3_0006 & 13.67 & 6.09 & 0.03 & 0.02 & rozier/.../P0.7N1L40_6 & 2.24 & 455.67 & 683.50 \\
\hline S3_0007 & 16.64 & 16.66 & 16.46 & 0.63 & $\operatorname{trp} / \mathrm{N} 12 \mathrm{y} / 36-12-0-36-3-0-200003$ & 1.00 & 1.01 & 26.41 \\
\hline S3_0011 & 17.53 & 17.24 & 17.18 & 1.13 & $\operatorname{trp} / \mathrm{N} 12 \mathrm{y} / 36-12-0-36-3-0-200004$ & 1.02 & 1.02 & 15.51 \\
\hline S3_0012 & 16.61 & 7.96 & 1.01 & 0.02 & rozier/.../P0.5N1L30_5 & 2.09 & 16.45 & 830.50 \\
\hline S3_0024 & 117.23 & 48.52 & 46.12 & 45.89 & rozier/.../counterCarryLinear12 & 2.42 & 2.54 & 2.55 \\
\hline S3_0026 & 35.42 & 34.75 & 34.74 & 1.38 & $\operatorname{trp} / \mathrm{N} 12 \mathrm{y} / 36-12-0-36-3-0-200006$ & 1.02 & 1.02 & 25.67 \\
\hline S3_0029 & 48.33 & 48.98 & 48.51 & 0.06 & $\operatorname{trp} / \mathrm{N} 12 \mathrm{y} / 58-12-0-58-3-0-200004$ & 0.99 & 1.00 & 805.50 \\
\hline S3_0032 & 43.92 & 21.87 & 3.45 & 0.03 & rozier/.../P0.333...N1L60_7 & 2.01 & 12.73 & 1464.00 \\
\hline S3_0033 & 128.02 & 48.54 & 44.42 & 42.58 & rozier/.../counterCarry 12 & 2.64 & 2.88 & 3.01 \\
\hline -0034 & 56.69 & 55.40 & 56.92 & 0.07 & $\operatorname{trp} / \mathrm{N} 12 \mathrm{y} / 46-12-0-46-3-0-200003$ & 1.02 & 1.00 & 809.86 \\
\hline S3_0038 & 75.14 & 73.54 & 73.44 & 37.59 & $\operatorname{trp} / \mathrm{N} 12 \mathrm{y} / 36-12-0-36-3-0-200005$ & 1.02 & 1.02 & 2.00 \\
\hline S3_0042 & 94.67 & 23.75 & 0.08 & 0.02 & rozier/.../P0.5N1L80_4 & 3.99 & 1183.38 & 4733.50 \\
\hline S3_0043 & 115.33 & 114.48 & 114.75 & 0.04 & $\operatorname{trp} / \mathrm{N} 12 \mathrm{y} / 66-12-0-66-3-0-200002$ & 1.01 & 1.01 & 2883.25 \\
\hline S3_0044 & 76.23 & 33.41 & 0.01 & 0.02 & rozier/...P0.95N1L80_1 & 2.28 & 7623.00 & 3811.50 \\
\hline
\end{tabular}

Table 1: Speed of U3/S3 formulas with split_depth of 18 and divided into 1, 2, 8, or 88 parallel jobs.

full implementation is available at https://github.com/gmatht/leviathan, along with links to raw benchmark data.

Despite its simplicity, this approach has a number of advantages. Each job is a simple single threaded task, avoiding the potential for "Heisenbugs" that can arise from multi-threading and other forms of nondeterministic parallelism. Since each job does not need to communicate with other jobs, we can easily run jobs on different cloud instances. For example, the largest single instance available on Amazon is the $128 \mathrm{vCPU} \mathrm{m} 4.16 \mathrm{xlarge}$ with 64 cores. In this paper we will discuss distributing tasks over more cores than available on a single EC2 instance. By measuring the amount of time each vertex takes to process, we can estimate the amount of time a formula would take to process if we had used a different number of cores. We can reproduce satisfiability results easily, including time required, on a single core by re-running the job that determined the formula was satisfiable. (Likewise, to demonstrate that the algorithm is slow for a known unsatisfiable formula, we only need to show that one job is slow.)

\section{Benchmarks}

We consider the widely used [1, 10] LTL benchmark sets: acacia, alaska, anzu, forobots, rozier, schuppan, and trp. We identify the formulas by filename, and include them in our fork of leviathan at https://github.com/gmatht/leviathan/tree/master/tests.

We conducted a preliminary study on these benchmarks, running them locally on an i7-4790 CPU @ 3.60GHz running an Ubuntu 16.04 VirtualBox under Windows 7 using default settings (that is, without the --release switch, which increased performance roughly ten fold). Based on this study we divided 


\begin{tabular}{|c|c|c|c|c|c|c|c|c|c|c|c|}
\hline \multicolumn{6}{|c|}{ split_depth $=18$} & \multicolumn{6}{|c|}{ split_depth=20 } \\
\hline jobs & set & mean & median & $\min$ & $\max$ & jobs & set & mean & median & $\min$ & $\max$ \\
\hline 2 & U2 & 1.97 & 1.98 & 1.92 & 2.00 & 2 & U2 & 1.83 & 1.84 & 1.70 & 1.91 \\
\hline 8 & $\mathrm{U} 2$ & 4.71 & 4.60 & 4.42 & 5.24 & 8 & $\mathrm{U} 2$ & 5.16 & 4.76 & 4.00 & 7.10 \\
\hline 32 & $\mathrm{U} 2$ & 15.14 & 15.34 & 11.50 & 17.91 & 32 & $\mathrm{U} 2$ & 12.47 & 13.52 & 7.93 & 16.41 \\
\hline 88 & $\mathrm{U} 2$ & 22.94 & 21.39 & 13.60 & 34.03 & 88 & $\mathrm{U} 2$ & 18.14 & 19.84 & 8.52 & 25.05 \\
\hline 2 & U3 & 2.04 & 2.03 & 1.98 & 2.19 & 2 & U3 & 1.99 & 2.00 & 1.85 & 2.05 \\
\hline 8 & $\mathrm{U} 3$ & 6.19 & 6.12 & 5.07 & 7.13 & 8 & U3 & 6.80 & 7.24 & 5.17 & 8.42 \\
\hline 32 & U3 & 20.24 & 20.12 & 17.25 & 23.31 & 32 & U3 & 19.93 & 20.29 & 18.20 & 21.55 \\
\hline 88 & U3 & 44.17 & 45.44 & 32.35 & 51.00 & 88 & U3 & 44.92 & 45.27 & 38.37 & 48.06 \\
\hline 2 & S2 & 1.08 & 1.01 & 0.98 & 2.07 & 2 & S2 & 1.02 & 0.98 & 0.84 & 1.95 \\
\hline 8 & $\mathrm{~S} 2$ & 1.50 & 1.01 & 0.81 & 12.44 & 8 & $\mathrm{~S} 2$ & 1.49 & 1.01 & 0.82 & 10.25 \\
\hline 32 & $\mathrm{~S} 2$ & 4.65 & 1.01 & 0.90 & 90.50 & 32 & $\mathrm{~S} 2$ & 3.90 & 1.01 & 0.75 & 67.88 \\
\hline 88 & $\mathrm{~S} 2$ & 14.86 & 1.01 & 0.91 & 543.00 & 88 & $\mathrm{~S} 2$ & 8.02 & 1.01 & 0.59 & 181.00 \\
\hline 2 & S3 & 1.25 & 1.02 & 0.98 & 3.99 & 2 & S3 & 1.25 & 1.01 & 0.99 & 3.60 \\
\hline 8 & S3 & 207.45 & 1.02 & 0.96 & 7623.00 & 8 & $\mathrm{~S} 3$ & 202.07 & 1.02 & 0.91 & 7623.00 \\
\hline 32 & S3 & 449.40 & 1.02 & 0.69 & 9467.00 & 32 & $\mathrm{~S} 3$ & 449.78 & 1.02 & 0.96 & 9467.00 \\
\hline 88 & S3 & 358.44 & 1.04 & 0.89 & 4733.50 & 88 & $\mathrm{~S} 3$ & 267.30 & 1.02 & 0.93 & 4733.50 \\
\hline
\end{tabular}

Table 2: Speedup factor parallel algorithm over serial algorithm

these formulas into Unsatifiable and Satisfiable, and 4 categories of difficultly, from (0): problems which took between $10^{-1}$ to $10^{0}$ seconds to solve through to (3): problems which took between $10^{2}$ to $10^{3}$ seconds to solve. We exclude problems easier than (0) as being too easy to consider parallelising and problems harder the (3) as being too hard to benchmark effectively. This gave us 8 benchmark sets, U0-3 and S0-3. For the cloud benchmarks we used Amazon c4 instanceswith Intel Xeon E5-2666 v3 CPUs.

In this paper we will use the term vertices to refer to the nodes of the tableau, i.e. the end points of a branch. We will use Amazon's term instance to refer to a computational node, i.e. a shared memory machine with one or more CPUs.

LTL formulas are commonly run on StarExec (https://www.starexec.org/), but to easily scale to large numbers of CPUs we use Amazon Elastic Compute Cloud (EC2, https://aws . amazon . com/ ec2/). On EC2 we use the modern Compute Optimized (c4) instances with Intel Xeon E5-2666 v3 (Haswell) CPUs. The c4 instances use hyper-threading to provide twice as many vCPUs as CPUs, which slows each job by about $65 \%$. Overall each vCPU is roughly half as powerful as a single thread of the desktop i7. In this paper, we only run one job per two vCPUs on the instance. Although hyperthreading can provide more performance per physical CPU, this complicates the benchmarks. Benchmarks exploiting all the vCPUs are considered in the expanded version [8].

\subsection{Satisfiable formulas}

Our coarse grained parallelism does not assist in processing a single branch faster. Rather, it allows leviathan to process multiple branches at the same time. Broadly, there are two possibilities: either one of alternate branches finds a solution faster, or the tableau heuristics picked the best branch anyway. For this reason, with satisfiable formulas we do not expect the performance to scale smoothly. Adding additional CPUs may merely add a little overhead, or it may make an otherwise infeasible problem suddenly feasible, as in Table 1 . 


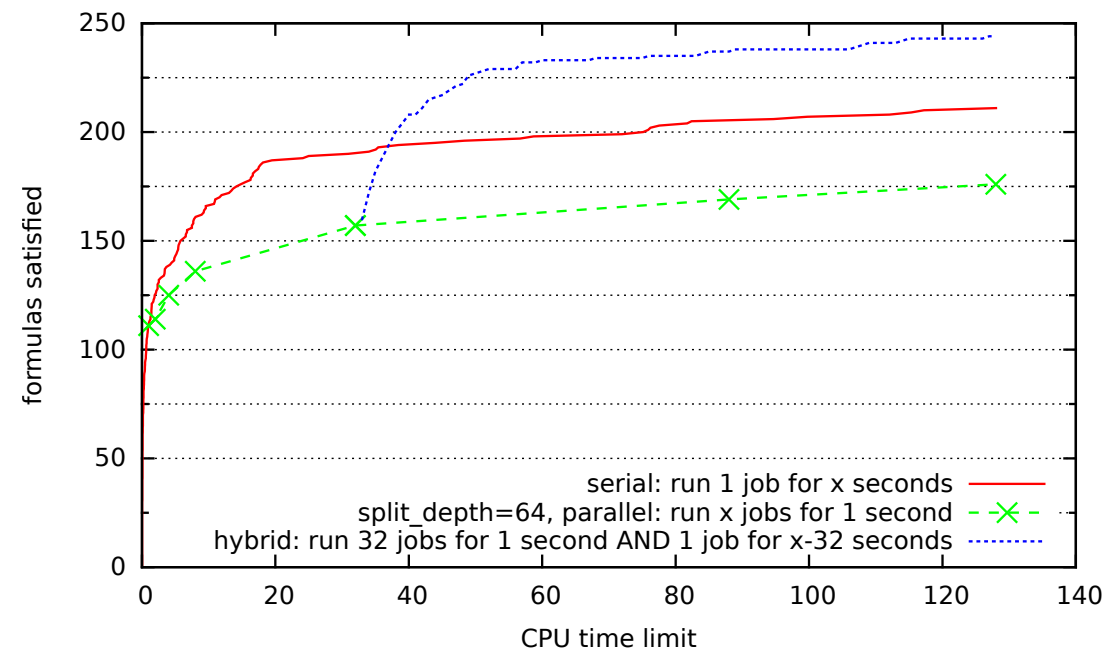

Figure 3: Number of formulas in the benchmark set shown to be satisfiable within $x$ CPU-seconds (e.g. number of CPUs allocated times time limit in seconds).

We consider the average performance over the benchmark sets in Table 2. We see that in each of the considered satisfiable cases, the median speedup factor is close to one; suggesting that parallel algorithm is not typically faster than the serial algorithm. On the other hand, the mean speedup is impressive.

The difference between the median and the mean is explained due the to fact that there is not limit to how much faster the parallel algorithm can be. If the serial algorithm finds a model, then at least one of the parallel jobs can find the model just as easily. On the other hand if the serial algorithm guesses wrongly as to which path leads to a model, it can perform very poorly. If one of the parallel jobs picks a better path, it could solve the problem almost instantly.

Given that the speedup on satisfiable formulas as we increase the number of CPUs is not smooth, we may instead consider the number of formulas that can be shown to be satisfiable within a single second. In Figure 3 we present the total number of formulas in the benchmark sets considered that can be shown to be satisfiable. A split_depth of 64 was identified as being effective for showing satisfiability during the pilot study [8] and was used in this graph. Increasing the number of jobs increases the number of formulas that are shown to be satisfiable, but not as quickly as increasing the number of seconds allowed. When limiting the serial algorithm to 15 seconds per formula, it can show 176 of the benchmark formulas are satisfiable, the same as the parallel algorithm can when limited to 128 jobs and 1 second. We see that the parallel algorithm is faster but is not as an efficient use of CPU time. On the other hand, the problems it finds hard are different to those the serial algorithm finds hard, so dividing a fixed CPU time budget between the serial and parallel algorithms can be more efficient than the original serial algorithm alone.

\subsection{Unsatisfiable formulas}

For unsatisfiable formulas the whole tableau must be constructed. Unlike satisfiable formulas, the amount of work will not be effected by the parallelisation changing the search order. For unsatisfiable formulas we expect a smoother increase in performance as we increase the number of jobs. We see in Table 1 that for unsatisfiable formulas more jobs consistently provides more performance. We see that out of this benchmark set the more challenging formulas got close to linear improvement in performance when 

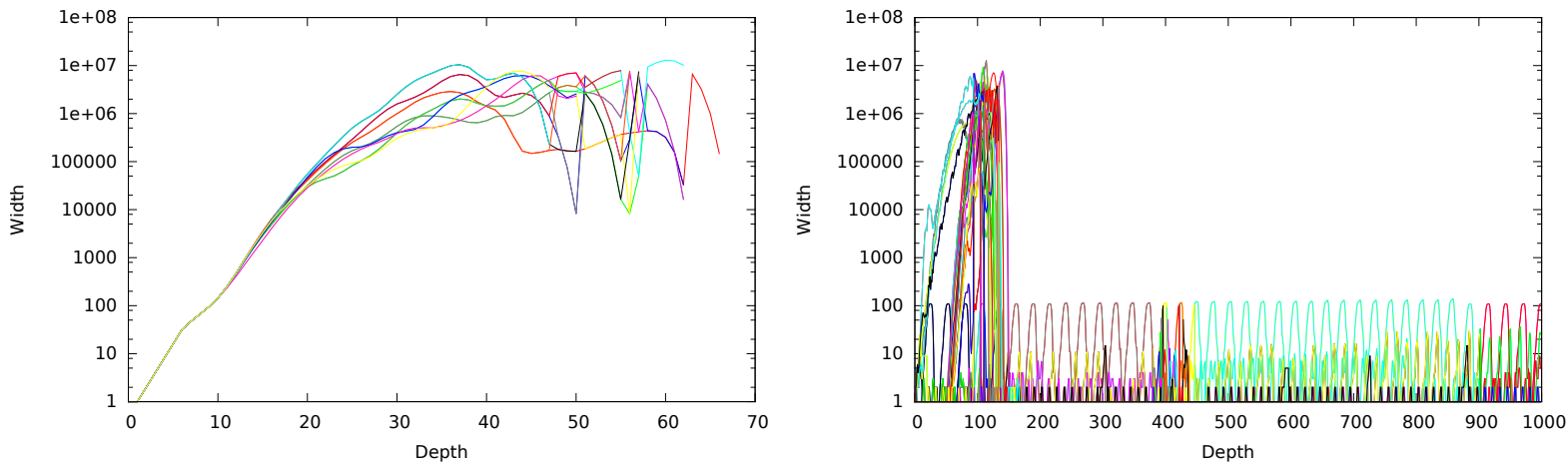

Figure 4: Depth vs Width of tableaux for unsatisfiable (left: U3) vs satisfiable (right: S3) formulas. Based on tableau constructed by serial version of leviathan. Each line describes the width of the tableau for a different input formula. The width is computed at the time the algorithm terminates, if the formula is satisfiable this may be before the full tableau is constructed.

increasing from 1 job to 88 jobs. These formulas were primarily N5x formulas from the trp set of randomly generated formulas.

Although tableaux are generally less useful for showing unsatisfiability, the pltl graph ${ }^{1}$ tableau based on the Schwendimann [19] technique is effective for showing unsatisfiability of N5x formulas. The pltl tableau took 21.7 seconds to show unsatisfiability of N5x formulas compared with 14.4 for the fastest technique, putting pltl in second place. Thus parallelising tableau for showing unsatisfiability of N5x formulas may lead to practical performance improvements in this area. Due to the interdependence of branches, parallelising pltl will not be as easy as parallelising leviathan, however.

\section{Analysis}

It this section we will discuss why some formulas are hard to parallelise, in context of the shape of the tableaux, and give an intuition of the shape of the tableau to better understand split_depth's effect.

\subsection{Depth and Width}

We consider the problem of finding the ideal split_depth, and explain why we chose a default of 18 .

To begin with, let us consider the width of the tableau. This is presented in Figure 4 . In the case of unsatisfiable tableaux, the width puts an upper bound on the amount of parallelization that can occur. Fortunately, for the challenging problems in the set U3, that is quite large. We see in Figure 4 that the depth of the tableau peaks around 1-10 million for each of the formulas. If we hope to utilize $n$ CPUs, we must pick a split_depth that provides at least that width. We may wish to have a greater depth, in the hope that if many vertices are assigned to a single job, that the harder and easier vertices will average out to provide a roughly equal amount of work in each vertex.

Increasing the split_depth also increases overhead. In our algorithm, all cores have to compute all vertices shallower than split_depth. In Table 3 we investigate how this overhead grows with increases in split_depth. The overhead of 1.077 seconds for U3_007 at a split_depth 25 is trivial for a

\footnotetext{
${ }^{1}$ Available, (1) of http://users.cecs.anu.edu.au/ rpg/PLTLProvers/
} 


\begin{tabular}{|l|c|c|c|c|c|c|c|c|c|c|c|}
\cline { 2 - 11 } \multicolumn{1}{c|}{} & \multicolumn{10}{c|}{ split_depth } \\
\hline Name & 15 & 16 & 17 & 18 & 19 & 20 & 21 & 22 & 23 & 24 & 25 \\
\hline U1_001 & 0.001 & 0.002 & 0.002 & 0.002 & 0.003 & 0.003 & 0.004 & 0.004 & 0.005 & 0.007 & 0.008 \\
U2_001 & 0.006 & 0.010 & 0.017 & 0.030 & 0.050 & 0.080 & 0.127 & 0.184 & 0.251 & 0.338 & 0.425 \\
U3_001 & 0.007 & 0.012 & 0.019 & 0.033 & 0.054 & 0.084 & 0.134 & 0.205 & 0.303 & 0.465 & 0.657 \\
U3_002 & 0.006 & 0.011 & 0.017 & 0.028 & 0.043 & 0.066 & 0.106 & 0.159 & 0.236 & 0.353 & 0.489 \\
U3_003 & 0.007 & 0.011 & 0.019 & 0.033 & 0.055 & 0.089 & 0.144 & 0.218 & 0.324 & 0.489 & 0.703 \\
U3_004 & 0.007 & 0.010 & 0.016 & 0.027 & 0.043 & 0.067 & 0.102 & 0.139 & 0.177 & 0.228 & 0.288 \\
U3_005 & 0.006 & 0.010 & 0.017 & 0.029 & 0.049 & 0.082 & 0.143 & 0.231 & 0.362 & 0.529 & 0.698 \\
U3_006 & 0.006 & 0.008 & 0.013 & 0.023 & 0.037 & 0.058 & 0.094 & 0.140 & 0.204 & 0.301 & 0.417 \\
U3_007 & 0.006 & 0.011 & 0.018 & 0.033 & 0.056 & 0.096 & 0.166 & 0.283 & 0.427 & 0.697 & 1.077 \\
U3_008 & 0.006 & 0.011 & 0.018 & 0.030 & 0.046 & 0.071 & 0.106 & 0.150 & 0.205 & 0.280 & 0.353 \\
\hline
\end{tabular}

Table 3: Total overhead of split_depth over all U0-U3 (unsatisfiable) formulas, measured in seconds taken by Job 0 (no vertices assigned to job)

\begin{tabular}{|l|r|r|r|r|r|r|r|r|}
\cline { 2 - 9 } \multicolumn{1}{c|}{} & \multicolumn{3}{c|}{ Raw Estimate } & \multicolumn{4}{c|}{ Actual time } \\
\hline Name & \multicolumn{1}{c|}{ job 1 } & job 2 & \multicolumn{1}{|c|}{ job 3 } & \multicolumn{1}{|c|}{ job 4 } & job 1 & job 2 & job 3 & \multicolumn{1}{c|}{ job4 } \\
\hline U3_0001 & 6.27 & 6.62 & 6.15 & 6.46 & 6.32 & 6.33 & 6.21 & 6.19 \\
U3_0002 & 9.14 & 9.14 & 6.78 & 6.65 & 6.65 & 6.66 & 6.87 & 6.73 \\
U3_0003 & 11.92 & 11.87 & 11.69 & 12.32 & 12.18 & 11.84 & 11.97 & 12.12 \\
U3_0004 & 12.94 & 13.01 & 13.20 & 12.43 & 12.80 & 13.20 & 13.08 & 12.63 \\
U3_0005 & 14.19 & 13.93 & 13.98 & 13.81 & 14.23 & 14.25 & 14.29 & 14.10 \\
U3_0006 & 17.40 & 19.03 & 18.30 & 18.26 & 18.08 & 19.45 & 19.01 & 18.61 \\
U3_0007 & 20.66 & 20.06 & 20.02 & 21.04 & 21.40 & 20.65 & 20.76 & 21.65 \\
U3_0008 & 30.27 & 29.17 & 30.22 & 26.44 & 30.89 & 29.86 & 30.68 & 27.02 \\
\hline
\end{tabular}

Table 4: Time taken by to process each formula, divided into four jobs at depth 17, using the "to_job" algorithm to assign vertices to jobs. Left is the raw estimate in seconds based on the number of time taken processing vertices in the single threaded case.

small number of vertices, since the total time is about 80 seconds. However, we are interested in the capability of tableaux to be massively parallelised, and the overhead becomes significant over 100 jobs, and guarantees that no matter how many jobs are used, the run time could never be brought down to 1 second with a split_depth of 25 . We will estimate how long the parallel tasks will take by recording how long it took the serial task to process each vertex and its children. We can determine which vertex would be assigned to which job, add up the amount of time taken to process all vertices assigned to that job. This allows us to estimate the length of each job, the amount of time taken to show unsatisfiability is roughly the length of the longest job.

We now consider what split_depth is a reasonable default in Table 5 . We see that, depending on the number of jobs, 18-20 is reasonable for the unsatisfiable formulas considered.

\subsection{When parallelisation does not help}

The difficulty in parallelising the other rows of Table 1 stems from the narrow width of the tableau. Many formulas only had a width of one, and in other cases the width of the tableau continually returned to one, 


\begin{tabular}{|l|r|r|r|r|r|r|r|r|r|r|c|}
\cline { 2 - 11 } \multicolumn{1}{c|}{} & \multicolumn{10}{|c|}{1 split_depth } \\
\hline jobs & \multicolumn{1}{|c|}{14} & \multicolumn{1}{c|}{15} & \multicolumn{1}{|c|}{17} & \multicolumn{1}{c|}{18} & \multicolumn{1}{c|}{19} & \multicolumn{1}{c|}{20} & \multicolumn{1}{c|}{21} & \multicolumn{1}{c|}{22} & \multicolumn{1}{c|}{23} & \multicolumn{1}{c|}{24} \\
\hline 1 & 527.0 & 527.0 & 527.1 & 527.1 & 527.3 & 527.5 & 527.9 & 528.6 & 529.5 & 527.5 & 528.0 \\
2 & 265.5 & 268.8 & 270.3 & 270.4 & 267.2 & 270.8 & 268.9 & 267.7 & 268.5 & 267.6 & 268.4 \\
4 & 142.2 & 137.4 & 140.0 & 139.4 & 137.7 & 138.3 & 136.6 & 137.5 & 138.2 & 136.6 & 138.8 \\
8 & 74.6 & 73.2 & 73.4 & 71.4 & 73.1 & 72.9 & 71.0 & 71.9 & 74.0 & 72.2 & 73.3 \\
16 & 43.1 & 41.4 & 39.8 & 39.0 & 37.9 & 37.9 & 38.6 & 39.4 & 40.4 & 39.3 & 40.9 \\
32 & 25.3 & 22.7 & 21.5 & 21.6 & 21.0 & 21.6 & 21.9 & 21.9 & 23.4 & 22.7 & 24.3 \\
64 & 14.9 & 14.2 & 12.7 & 12.6 & 13.3 & 12.7 & 12.5 & 13.4 & 14.8 & 14.3 & 16.1 \\
88 & 12.2 & 10.5 & 10.8 & 10.8 & 11.2 & 9.8 & 10.2 & 11.4 & 12.3 & 12.1 & 13.4 \\
128 & 9.5 & 8.7 & 8.4 & 8.3 & 7.6 & 8.1 & 8.6 & 9.4 & 10.5 & 10.1 & 11.5 \\
\hline
\end{tabular}

Table 5: Estimated time to solve all the unsatisfiable formulas in sets U0-U3 performance given various number of parallel jobs and choices of split_depth

indicating that only one branch was particularly difficult (see Figure 4 for a visualisation).

Brute force parallelisation does not always substitute for finding a more appropriate algorithm. For example, pltl graph takes just under 4 seconds to show that trp/N5x/12/pltl-5-0-12-3-0-200003 is unsatisfiable. With a split depth of 26 overhead was 3.9-4.1s, so clearly we cannot beat pltl graph on the problem using a split depth greater than 26 . We have 224984 vertices at depth 26 , so we could in principle have up to 224984 jobs running at the same time. However, Task 2 out of 224984 did not complete within minutes, let alone seconds.

\section{Parallel Tableaux as Contributor to Portfolio Reasoners}

In this section we consider what part a parallel tableau could play as part of a combined tool which uses several more basic LTL satisfiability techniques as alternatives in parallel on a given input. For example, the portfolio tool POLSAT [10], makes use of a range of the best tableaux, resolution, automata and symbolic tools, all set off in parallel on the input formula.

Also published in [10] is a comprehensive list of benchmarking a variety of best performing reasoners across the families of Schuppan benchmarks (http://www.schuppan.de/viktor/atva11/). From this we can see that the tableau tools are often the fastest type of tools across satisfiable formulas but rarely on unsatisfiable formulas.

From the results in Sections 4.1 and 4.2 above we also know that parallelising suitable tableaux (such as the Prune-rule-based Leviathan approach) can lead to (1) easily gained impressive speed-ups on some satisfiable formulas; (2) not much of a speed-up on many satisfiable formulas; and (3) solid speed-ups on many unsatisfiable formulas. Together these observations suggest that it is worth incorporating a parallel tableau approach in these portfolio approaches.

We should also mention the very recent new SAT-based explicit temporal reasoner presented in [12] which uses the technique of "semantic splitting" [14] like state-of-the-art propositional SAT solvers, instead of the syntactic splitting seen in tableaux approaches. This technique is showing impressively fast results via the implementation Aalta v2.0. The timings presented in the appendix of [12] show that this is much faster on most of the benchmarks than LS4 [20], TRP++ [7], NuXmv-BMCINC [6], and the tableaux/automata reasoner Aalta v1.2 from [9]. Aalta 2.0 does seem to perform significantly faster than the other tools almost across the benchmarks so it will be interesting to see in future work whether the 
speed-ups that we are seeing in parallel tableaux techniques can challenge this in any way.

\subsection{Effectiveness}

We are most interested in comparing the performance of the parallel tableau to other tableaux. The tableaux pltl graph and pltl tree are available from http://users.cecs.anu.edu.au/ rpg/ software.html. These are particularly interesting because pltl graph is widely considered the most promising LTL tableau, and pltl tree is a tree tableau similar to leviathan.

To evaluate the intuition that a parallel leviathan would be a useful contribution to a portfolio reasoner we generated a large number of new pseudo random formulas. Although the trp set contains randomly generated formulas, the set of $\operatorname{trp}$ formulas that are not already trival to solve with existing serial tableau techniques is too small to form a meaningful benchmark set.

We adapt our existing random generator for Full Computation Tree Logic (CTL*) [15] formulas to LTL, resulting in the following recursive procedure: we generate a formula of length $n$ recursively as follows. For $n=1$ we pick an atomic proposition at random from $\{p, q\}$; for $n=2$ we pick at random a unary operator from $\{\neg, X, F, G\}$ and an atom from $\{p, q\}$. For $n>2$ we first choose to start with a binary operator or unary operator with equal probability. Out of $10^{6}$ formulas of length 50, we eliminated all formulas that could be reasoned with by pltl graph or pltl tree within a second. This left 2779 formulas. Eliminating the formulas that could not be solved by serial leviathan left 314 unsolved formulas. Using 8 jobs with a split_depth of 18 or 20 reduced the number of unsolved formulas to 300 or 299 respectively, a slight improvement over the serial case.

PolSAT is presently available from http://lab301.cn/home/pages/lijianwen/. We were not able to get the bundled version of altaa to compile, so we replaced it with version 2. It was difficult to find formulas of length 50 that could not already be solved within 1 second by PolSAT, so we increased the formula length to 400 . We found 23460 formulas that could not be solved by PolSAT in 1 second. Out of these, leviathan could solve 100 in one second (0.5\%). We took 2000 formulas that had not been solved in one second by either the serial leviathan tableau or PolSAT. We then put those 2000 formulas into the parallel algorithm running on the cloud with 16 vCPUs ( 8 physical CPUs), using a split_depth of 20. The parallel algorithm was able to show that $6(0.3 \%)$ of these formulas were satisfiable, and was not able to show that any were unsatisfiable. The hardest of these 6 formulas took PolSAT 28 seconds.

In summary, although parallelising a tableau algorithm can provide dramatic speedups, novel algorithms have more to offer than parallelisation of existing algorithms.

\section{Conclusions and Future Work}

We see that parallelising tableaux can indeed be easy. Though our parallelisation technique is simple, its greatest weakness is simply that a highly parallel tableau is no substitute for a tableau optimised to the class of LTL formulas of interest. Thus, the shortage of prior parallel tableau may be explained by the vast potential for performance improvements in serial algorithms. We have demonstrated a significant speedups over the serial version. These speedups are not guaranteed for satisfiable formulas, but are frequently much better than linear. For unsatisfiable formulas, the speedups are reliable, but sublinear. When it is reasonable to use a large number of CPUs, this provides a significant incremental improvement in the amount of formulas considered that can be reasoned about using tableau.

We have also shown that this simple approach is far from a panacea. In general, leviathan is highly effective at showing that a particular class of formulas is satisfiable, and parallelisation broadens the 
class of formulas it can show is satisfiable. Even massive parallelisation would not make leviathan beat techniques optimised for showing unsatisfiability at their own game.

It is not clear that more sophisticated parallelisation techniques would help. Before settling on the present algorithm, we tried starting a new process for each branch at split_depth so that we could dynamically assign branches to CPUs as they became idle. The overhead of starting new processes turned out not to be worthwhile. One could use a POSIX "fork()" to avoid this overhead, but this would not scale naturally past a single machine. One could instead serialise the state of the tableau and transmit it to a new machine, but this would be invasive as leviathan has various types of internal state.

Since we have had close to linear growth in performance in unsatisfiable formulas, there is little to gain here by improving parallelisation. By contrast switching to a tableau better at showing unsatisfiability such as pltl graph gave massive improvements in of some formulas. Simply improving the parallelisation of leviathan will not bring its performance close to pltl graph on those formulas.

This simple parallelisation technique is more practically useful for making leviathan even more effective at showing satisfiability than it already is. In the case of the more challenging satisfiability formulas in the $\mathrm{S} 3$ set and a split_depth of 20 , there were no cases a job terminating before the formula was shown to be satisfiable, so there were no idle CPUs to move work to. Thus, it is hard to justify the complexity of more advanced parallelisation techniques. This complexity would be better spent on more advanced heuristics and parallelising more reasoning techniques. We have seen that adding a parallel version of leviathan would only improve PolSAT slightly. We would expect a greater increase from parallelising the more optimised pltl graph tableau, a more challenging task.

The unsatisfiable formulas studied had a fairly similar exponential growth in up to depth 20 . This provided enough width for a high degree of parallelism over these formulas at the fixed split_depth of 18. By contrast satisfiable formulas were less predictable as to where the ideal split_depth was, frequently 64 was better than 18 for satisfiable formulas. There would be some benefit to automatically detecting the ideal split_depth, however for a number of formulas no fixed depth would suit and a different method of parallelisation would need to be used.

Acknowledgements. This research was funded partially by the Australian Government through the Australian Research Council. (project DP140103365)

\section{References}

[1] Matteo Bertello, Nicola Gigante, Angelo Montanari \& Mark Reynolds (2016): Leviathan: A New LTL Satisfiability Checking Tool Based on a One-Pass Tree-Shaped Tableau. In Subbarao Kambhampati, editor: Proceedings of the Twenty-Fifth International Joint Conference on Artificial Intelligence, IJCAI 2016, New York, NY, USA, 9-15 July 2016, IJCAI/AAAI Press, pp. 950-956. Available at http://www.ijcai.org/ Abstract/16/139.

[2] Alessandro Cimatti, Edmund M. Clarke, Enrico Giunchiglia, Fausto Giunchiglia, Marco Pistore, Marco Roveri, Roberto Sebastiani \& Armando Tacchella (2002): NuSMV 2: An OpenSource Tool for Symbolic Model Checking. In Ed Brinksma \& Kim Guldstrand Larsen, editors: Computer Aided Verification, 14th International Conference, CAV 2002, Copenhagen, Denmark, July 27-31, 2002, Proceedings, Lecture Notes in Computer Science 2404, Springer, pp. 359-364, doi:10.1007/3-540-45657-0_29

[3] Ian T. Foster (1995): Designing and building parallel programs - concepts and tools for parallel software engineering. Addison-Wesley.

[4] Oliver Friedmann, Markus Latte \& Martin Lange (2010): A Decision Procedure for CTL* Based on Tableaux and Automata. In Jürgen Giesl \& Reiner Hähnle, editors: IJCAR, 6173, Springer, pp. 331-345, doi:10.1007/978-3-642-14203-1_28. 
[5] Nicola Gigante, Angelo Montanari \& Mark Reynolds (2017): A One-Pass Tree-Shaped Tableau for LTL+Past. In Thomas Eiter \& David Sands, editors: 21st International Conference on Logic for Programming, Artificial Intelligence and Reasoning, LPAR-21, 7-12 May 2017, Maun, Botswana, LNCS 46, Springer, pp. 456-473.

[6] K. Heljanko, T. Junttila \& T. Latvala (2005): Incremental and complete bounded model checking for full PLTL, pp. 98-111. Springer International Publishing, doi:10.1007/11513988_10

[7] Ullrich Hustadt \& Boris Konev (2003): TRP++2.0: A Temporal Resolution Prover. In Franz Baader, editor: Automated Deduction - CADE-19, 19th International Conference on Automated Deduction Miami Beach, FL, USA, July 28 - August 2, 2003, Proceedings, Lecture Notes in Computer Science 2741, Springer, pp. 274-278, doi:10.1007/978-3-540-45085-6_21

[8] Mark Reynolds John McCabe-Dansted (2017): LTL in the cloud (expanded version). http://staffhome. ecm.uwa.edu.au/ 00061811/papers/parallel.pdf

[9] Jianwen Li, Geguang Pu, Lijun Zhang, Moshe Y. Vardi \& Jifeng He (2014): Fast LTL Satisfiability Checking by SAT Solvers. CoRR abs/1401.5677. Available at http://arxiv.org/abs/1401.5677

[10] Jianwen Li, Geguang Pu, Lijun Zhang, Yinbo Yao, Moshe Y. Vardi \& Jifeng He (2013): Polsat: A Portfolio LTL Satisfiability Solver. CoRR abs/1311.1602. Available at http://arxiv.org/abs/1311.1602

[11] Jianwen Li, Yinbo Yao, Geguang Pu, Lijun Zhang \& Jifeng He (2014): Aalta: an LTL satisfiability checker over Infinite/Finite traces. In Shing-Chi Cheung, Alessandro Orso \& Margaret-Anne D. Storey, editors: Proceedings of the 22nd ACM SIGSOFT International Symposium on Foundations of Software Engineering, (FSE-22), Hong Kong, China, November 16 - 22, 2014, ACM, pp. 731-734, doi $10.1145 / 2635868.2661669$

[12] Jianwen Li, Shufang Zhu, Geguang Pu \& Moshe Y. Vardi (2015): SAT-Based Explicit LTL Reasoning, pp. 209-224. Springer International Publishing, Cham, doi:10.1007/978-3-319-26287-1_13.

[13] C. Limongelli, A. Orlandini \& V. Poggioni (2003): A Parallel Computation Technique for Linear Time Logic Tableaux. In: Tableaux 2003, Position Papers and Tutorials. Available at http://limongelli.dia. uniroma3.it/papers/tab2003.ps.

[14] S. Malik \& L. Zhang (2009): Boolean satisfiability from theoretical hardness to practical success. Commun. ACM 52(8), pp. 76 -82, doi:10.1145/1536616.1536637.

[15] John C. MCabe-Dansted \& Mark Reynolds (2017): Rewrite rules for CTL*. Journal of Applied Logic 21, pp. 24 - 56, doi $10.1016 / \mathrm{j}$.jal.2016.12.003

[16] A. Pnueli (1977): The temporal logic of programs. In: Proceedings of the Eighteenth Symposium on Foundations of Computer Science, pp. 46-57, doi:10.1109/SFCS.1977.32. Providence, RI.

[17] Mark Reynolds (2016): A New Rule for LTL Tableaux. In Domenico Cantone \& Giorgio Delzanno, editors: Proceedings of the Seventh International Symposium on Games, Automata, Logics and Formal Verification, GandALF 2016, Catania, Italy, 14-16 September 2016., EPTCS 226, pp. 287-301, doi $10.4204 /$ EPTCS.226.20

[18] Viktor Schuppan \& Luthfi Darmawan (2011): Evaluating LTL Satisfiability Solvers. In Tevfik Bultan \& Pao-Ann Hsiung, editors: ATVA'11, Lecture Notes in Computer Science 6996, Springer, pp. 397-413, doi $10.1007 / 978-3-642-24372-1 \_28$

[19] Stefan Schwendimann (1998): A New One-Pass Tableau Calculus for PLTL. In Harrie C. M. de Swart, editor: Automated Reasoning with Analytic Tableaux and Related Methods, International Conference, TABLEAUX '98, Oisterwijk, The Netherlands, May 5-8, 1998, Proceedings, Lecture Notes in Computer Science 1397, Springer, pp. 277-292, doi:10.1007/3-540-69778-0_28.

[20] Martin Suda \& Christoph Weidenbach (2012): A PLTL-Prover Based on Labelled Superposition with Partial Model Guidance. In Bernhard Gramlich, Dale Miller \& Uli Sattler, editors: Automated Reasoning - 6th International Joint Conference, IJCAR 2012, Manchester, UK, June 26-29, 2012. Proceedings, Lecture Notes in Computer Science 7364, Springer, pp. 537-543, doi:10.1007/978-3-642-31365-3_42

[21] O. Tange (2011): GNU Parallel - The Command-Line Power Tool. ;login: The USENIX Magazine 36(1), pp. 42-47. Available at http://www.gnu.org/s/parallel. 